\title{
On Collisionless Ion and Electron Populations in the Magnetic Nozzle Experiment (MNX)
}

\author{
Samuel A. Cohen, Xuan Sun, Nathaniel M. Ferraro, Earl E. Scime, Mahmood Miah, Sy Stange, \\ Nicholas S. Siefert, and Robert F. Boivin
}

\begin{abstract}
The Magnetic Nozzle Experiment (MNX) is a linear magnetized helicon-heated plasma device, with applications to advanced spacecraft-propulsion methods and solar-corona physics. This paper reviews ion and electron energy distributions measured in MNX with laser-induced fluorescence (LIF) and probes, respectively. Ions, cold and highly collisional in the main $\mathrm{MNX}$ region, are accelerated along a uniform magnetic field to sonic then supersonic speeds as they exit the main region through either mechanical or magnetic apertures. A sharp decrease in density downstream of the aperture(s) helps effect a transition from collisional to collisionless plasma. The electrons in the downstream region have an average energy somewhat higher than that in the main region. From LIF ion-velocity measurements, we find upstream of the aperture a presheath of strength $\Delta \phi_{p s}={ }_{m r} T_{e}$, where ${ }_{m r} T_{e}$ is the electron temperature in the main region, and length $\sim 3 \mathrm{~cm}$, comparable to the ion-neutral mean-free-path; immediately downstream of the aperture is an electrostatic double layer of strength $\Delta \phi_{D L}=3-10{ }_{m r} T_{e}$ and length $0.3-0.6 \mathrm{~cm}, 30-600 \lambda_{D}$. The existence of a small, $\mathrm{ca} .0 .1 \%$, superthermal electron population with average energy $\sim 10{ }_{m r} T_{e}$ is inferred from considerations of spectroscopic line ratios, floating potentials, and Langmuir probe data. The superthermal electrons are suggested to be the source for the large $\Delta \phi_{D L}$.
\end{abstract}

Index Terms-Double layer, helicon, laser-induced-fluorescence (LIF), magnetic nozzle.

\section{INTRODUCTION}

H ELICON plasmas [1], [2] have been extensively studied in laboratories for over thirty years [3], [4] to elucidate the physics of wave propagation and plasma generation and to develop techniques for the plasma processing of materials, especially for the fabrication of semiconductor devices [5], [6] and for the destruction of toxic waste [7]. These applications are possible because the plasmas generated by the absorption of helicon waves are steady state, may reach densities of $n_{e}=10^{14} \mathrm{~cm}^{-3}$, and are of relatively low temperature, $T_{e}=1-10 \mathrm{eV}$, parameters that result in relatively high rates for surface modification

Manuscript received July 28, 2005; revised October 3, 2005. This work was supported in part by the U.S. Department of Energy under Contract DE-AC02-76-CHO-3073. The West Virginia University portion of this work was supported by the U.S. Department of Energy EPSCoR Laboratory Partnership Program under Grant ER45849.

S. A. Cohen, N. M. Ferraro, and M. Miah are with the Plasma Physics Laboratory, Princeton University, Princeton, NJ 08543 USA (e-mail: scohen@pppl. gov).

X. Sun and E. E. Scime are with the Physics Department, West Virginia University, Morgantown, WV 26506 USA.

S. Stange is with the University Research Program in Robotics, University of Michigan, Ann Arbor, MI 48109 USA.

N. S. Siefert is with the Air Force Research Laboratory, Wright-Patterson Air Force Base, Wright-Patterson Air Force Base, OH 45433-5543 USA.

R. F. Boivin is with the Physics Department, Auburn University, Auburn, AL 36849-5311 USA.

Digital Object Identifier 10.1109/TPS.2006.875846 and gas-phase ionization and decomposition at modest power consumption.

The good efficiency of helicon sources in producing dense plasmas [8]-[10] has brought attention to a new application, improving spacecraft-propulsion methods, particularly for missions to remote planets [11], [12]. For this application, high propellent (plasma) flow speeds and high directionality are desired for the rocket exhaust stream, to enable the spacecraft to reach high speeds [13], [14]. Collisionless plasmas are expected in the exhaust stream of advanced propulsion systems, in large part due to expansion of the plasma jet. For magnetized plasmas used for propulsion, collisionless processes must enable the plasma jet to detach from the rocket engine's magnetic field [15]. This is a subject of on-going research [16], [17]. As shown in this paper, some species are collisionless in the denser region, near the plasma source.

A figure-of-merit for propulsion is the specific impulse, $I_{s p} \equiv$ $v / g$, where $v$ is the directed flow velocity of the plasma and $g$ is the acceleration of gravity, $9.8 \mathrm{~m} / \mathrm{s}^{2}$. Chemical rockets achieve $I_{s p}$ as high as $500 \mathrm{~s}$; propulsion methods which employ plasma or ion beams have achieved $I_{s p}>10^{4} \mathrm{~s}$ [13]. (An $I_{s p}$ of 3000 $\mathrm{s}$, corresponding to an energy of $\sim 4.5 \mathrm{eV} / \mathrm{amu}$, is considered the minimum requirement for many remote-planet missions.)

Other yardsticks for advanced spacecraft-propulsion technologies include energy and fuel-utilization efficiencies and thrust. Too high an $I_{s p}$ gives less thrust per unit of power, hence limits on and control of $I_{s p}$ are required, with values set by the specific demands of each spacecraft mission and the capability of each spacecraft. In many advanced propulsion systems, energy efficiency will depend on the transport characteristics of collisionless plasmas. Another important factor determining energy efficiency is the energy "wasted" in ionization. To lower the ionization-energy penalty, it is advantageous to accelerate massive singly ionized atoms to higher energy than to accelerate light multiply charged ions to lower energy. The results described herein concentrate on argon plasmas-with occasional helium doping for spectroscopic diagnostic purposes - though xenon would offer an advantage in energy efficiency.

The question arises of how to accelerate argon ions to $180 \mathrm{eV}$, i.e., an $I_{s p}$ of $3000 \mathrm{~s}$. One promising approach is the use of ion cyclotron-resonance heating [18], [19]. Our experiments follow a different path made evident by the discovery of a natural phenomenon in high-density helicon plasmas, the formation of strong electrostatic double layers which accelerate ions into directed beams having supersonic speeds [16], [20]. Double layers have been observed in earlier low-density experiments [21]-[27] and recently [28], [29] in other helicon devices. This alternate path allows a small scale, low cost, relatively simple 


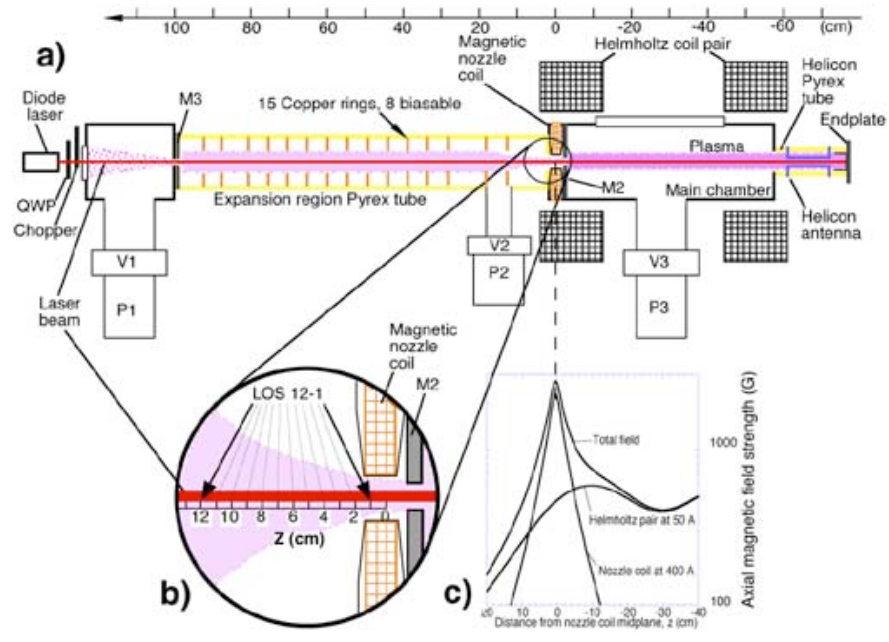

Fig. 1. Schematic of MNX. (a) Argon plasma is formed by absorption of helicon waves launched from a double-saddle antenna. Plasma flows through the main chamber along magnetic field fines created by a pair of Helmholtz coils. Plasma then flows through the aperture in M2 and the nozzle coil into the expansion region (ER). Beam of a diode laser is directed along the MNX axis. Scanning Langmuir probes are located in the center of main chamber and at $z=12,24$, and $70 \mathrm{~cm}$ in the ER. (b) LIF collection optics have 12 LOS intercepting axial points in the ER near the nozzle. Other LOS are in the main chamber. (c) On-axis axial magnetic field strength near the nozzle coil.

experimental facility to explore many physics issues of flowing collisionless plasmas.

In Section II we describe the experimental facility and the laser-induced-fluorescence (LIF), spectroscopic, and probe diagnostics. Sections III, IV, and V present ion and electron distributions and spectroscopic line ratios measured in MNX. Section VI summarizes the results and presents plans for future research.

\section{EXPERIMENTAL SETUP}

The experiments were performed in the Magnetic-NozzleExperiment device (MNX), see Fig. 1(a). Steady-state argon plasma is formed by the helicon technique. The antenna, a double-saddle design, is placed around a 4-cm-inner-diameter, 30-cm-long Pyrex tube; RF power, at $26.75 \mathrm{MHz}$, is provided to a T-type tank circuit with the antenna as inductor. The 4-cm-diameter plasma thus formed flows along the magnetic field formed by a Helmholtz-coil pair placed coaxially around the 45-cm-long, 20-cm-inner-diameter, stainless-steel main chamber (MC). An iron plug placed on the endplate of the 30-cm Pyrex tube helps shape the magnetic field inside the tube. (Several endplate designs have been used. The present one is a reentrant carbon-fiber-composite cup which reduces sputter-coating of the Pyrex tube with endplate material.) Each Helmholtz coil consists of 11 pancake coils which may be wired in any combination, allowing axial fields as high as 4 $\mathrm{kG}$ in the center of the main chamber, corresponding to 5.5 $\mathrm{kG}$ in the midplane of a Helmholtz coil. Plasma and device parameters of MNX are summarized in Table I.

The plasma exits the MC through the coaxial 2-cm-inner-diameter, 3-cm-long magnetic nozzle coil used to control the field gradient and mirror ratio. The maximum steady-state on-axis magnetic field the nozzle coil produces is $2.5 \mathrm{kG}$. Fig. 1(b) shows possible lines-of-sight for LIF collection optics used to view the plasma exiting the nozzle coil. Fig. 1(c) shows the axial field strength near the nozzle at a Helmholtz coil current of $50 \mathrm{~A}$ and nozzle current of $400 \mathrm{~A}$, typical of experimental conditions in this paper. The maximum current of both the Helmholtz and nozzle coils is $440 \mathrm{~A}$.

Exiting the nozzle coil, the plasma enters a 10-cm-inner-diameter, 100-cm-long Pyrex tube termed the expansion region (ER). The ER has 15 internal 4-cm-inner-diameter, 0.3-cm-thick coaxial copper rings, of which 8 may be electrically biased. Additionally, there are three metal discs, labeled endplate, $M 2$, and $M 3$ in Fig. 1(a), which may be electrically biased. (In the experiments described here, unless specificially noted otherwise, the endplate, M2, and M3 were left electrically floating. Biasing the endplate or M3 had little effect on the LIF results; connecting the endplate or M2 to ground required more RF power input to sustain the plasma.) M2 has an 0.24 to $1.0-\mathrm{cm}$-inner-diameter aperture hole which limits both the plasma and neutral gas flow into the ER. The axial location of M2 and the size of its aperture have been varied, to explore the roles of field gradients and mechanical apertures on double-layer (DL) parameters. In the absence of plasma and in conjunction with the ER pump, [P2, see Fig. 1(a)], the low conductance of M2 maintains up to a $\times 10$ lower pressure in the ER compared with the main chamber. Closing valves V1, V2, and/or V3, or leaking gas into the ER, allows controlled increase of the pressure in the ER. Pressures are measured in the main chamber and the ER by capacitance manometers with accuracies of \pm 0.1 and $\pm 0.001 \mathrm{mT}$, respectively.

At low Helmholtz fields, $B_{H}=200-1200 \mathrm{G}$, MNX operates stably in the "blue-core" helicon mode over a wide range of main-chamber pressures, from 0.4 to above $20 \mathrm{mT}$, at RF powers from 200 to over $2000 \mathrm{~W}$. The blue core, about $2 \mathrm{~cm}$ in diameter at $B_{H}=400 \mathrm{G}$, decreases in size as the field is increased, reaching $0.3 \mathrm{~cm}$ at $B_{H}=2.5 \mathrm{kG}$ and then increases in size. Most results described herein were obtained near the lowest main-chamber neutral pressure, to reduce collisional de-excitation of the $A r^{+*}$, the species necessary for the LIF signal.

The Langmuir probe in the main chamber is a single probe of cylindrical shape with radius $0.025 \mathrm{~cm}$. A 1.9-MHz low-pass filter is used to eliminate RF pickup. Radial scanning is accomplished by a pneumatic drive. Langmuir characteristics, over a voltage range from -140 to $+60 \mathrm{~V}$, are acquired at $10-100 \mathrm{~Hz}$, providing a spatial resolution as small as $1 \mathrm{~mm}$. Langmuir probe spatial scans in the main chamber, described in more detail in Section III, show that the plasmas achieved peak ion densities up to $5 \times 10^{13} \mathrm{~cm}^{-3}$ and electron temperatures in the range $2-10 \mathrm{eV}$. The line-averaged electron density is measured by a $170-\mathrm{GHz}$ interferometer directed across a major chord in the center of the main chamber. The interferometer was used to calibrate the probe-measured density. Langmuir probes in the ER are paddle-shaped single probes, coated with boron nitride on one side.

To permit measurement of the field-parallel $\mathrm{Ar}^{+*}$ velocity distribution in MNX, the elliptical-cross-section $(3.2 \times 1$ $\mathrm{mm}$ ) tuneable diode-laser beam is directed along the MNX magnetic axis. (To measure the perpendicular velocity in the 
TABLE I

Plasma AND DeVICE PARAMETERS OF MNX

\begin{tabular}{|c|c|c|}
\hline Parameters & main region & expansion region \\
\hline$B(\mathrm{kG})$ & $0.5-5.5$ & 8-0.02 \\
\hline $\mathrm{L}(\mathrm{cm})$ & 70 & 100 \\
\hline$n_{e}\left(10^{13} \mathrm{~cm}^{-3}\right)$ & $0.1-5$ & $0.1-10^{-4}$ \\
\hline$p_{n}(\mathrm{mT})$ & $0.4-20$ & $0.1-20$ \\
\hline $\mathrm{RF}$ heating power $(\mathrm{W})$ & $50-2000$ & \\
\hline$\omega_{p e}\left(10^{10} \mathrm{rad} / \mathrm{s}\right)$ & $5.6-40$ & $5.6-0.18$ \\
\hline$\omega_{c e}\left(10^{9} \mathrm{rad} / \mathrm{s}\right)$ & $9-100$ & $145-0.35$ \\
\hline$\omega_{c i}\left(10^{6} \mathrm{rad} / \mathrm{s}\right)$ & $0.12-1.3$ & $2-0.005$ \\
\hline Probe radius, $r_{p},(\mathrm{~cm})$ & 0.025 & 0.5 \\
\hline Plasma radius, $R,(\mathrm{~cm})$ & 2 & $0.1-4$ \\
\hline Debye length, $\lambda_{D},(\mathrm{~cm})$ & $2 \times 10^{-3}-1.5 \times 10^{-4}$ & $7 \times 10^{-2}-1.8 \times 10^{-3}$ \\
\hline Ion gyroradius, $\rho_{i},(\mathrm{~cm})$ & $1-0.025$ & $0.015-45$ \\
\hline Electron gyroradius, $\rho_{e},(\mathrm{~cm})$ & $1.3 \times 10^{-2}-6 \times 10^{-4}$ & $0.4-7 \times 10^{-4}$ \\
\hline$T_{e}(\mathrm{eV})$ & $2-10$ & $6-20$ \\
\hline$E_{e, \max }(\mathrm{eV})$ & $\geq 50$ & 220 \\
\hline$E_{i, \|}(\mathrm{eV})$ & $0.04-0.6$ ( 8 in presheath) & $0.06-80$ \\
\hline$T_{i, \|}(\mathrm{eV})$ & $0.03-0.4$ & $0.04-2$ \\
\hline$E_{i, \perp}(\mathrm{eV})$ & $0.04-0.9$ & \\
\hline$T_{i, \perp}(\mathrm{eV})$ & $0.03-0.6$ & \\
\hline$\lambda_{i i}(\mathrm{~cm})$ & $2 \times 10^{-5}-0.3$ & $2 \times 10^{-2}-7 \times 10^{3}$ \\
\hline$\lambda_{e e}(\mathrm{~cm})$ & $0.09-100$ & $35-10^{5}$ \\
\hline$\lambda_{e n}(\mathrm{~cm})$ & $0.3-15$ & $0.3-60$ \\
\hline$\lambda_{\text {in }}(\mathrm{cm})$ & $0.1-5$ & $0.1-20$ \\
\hline
\end{tabular}

main chamber, the laser beam was directed perpendicular to the magnetic field in the main chamber, not shown in Fig. 1). Optics to collect the fluorescence emission are located on both the main chamber and the expansion chamber. Main-chamber lines-of-sight (LOS) collect photons from a selectable $5 \mathrm{~mm}^{3}$ volume near the center of the chamber downstream to M2. Scanning optics on the ER allow LOSs which intercept the laser beam from $1 \mathrm{~cm}$ from the nozzle-coil midplane to 12 $\mathrm{cm}$ from its midplane, as well as beyond, see Fig. 1(b). LIF measurements in field gradients are relevant to the physics of the solar corona [30].

The laser used for LIF is a 1.5-MHz-bandwidth Sacher LaserTechnik diode laser, which may be coarse tuned in wavelength from 662 to $674 \mathrm{~nm}$, allowing excitation of the $668.614 \mathrm{~nm}$ (zero-field, vacuum) transition $3 d^{4} F_{7 / 2}-4 p^{4} D_{5 / 2}$ of $\mathrm{Ar}^{+*}$ [31], [32]. Finer tuning, over a maximum $0.4 \mathrm{~nm}$ wavelength range, is accomplished, manually or automatically, by changing the voltage on the laser's internal piezoelectric crystal. A photomultiplier with 1-nm transmission filter centered at $442.7 \mathrm{~nm}$ (vacuum) collects emission from the 442.7 $\mathrm{nm}$ fluorescence transition, $4 p^{4} D_{5 / 2}-4 s^{4} P_{3 / 2}$, with Einstein coefficient $A=9.856 \times 10^{7} \mathrm{~s}^{-1}$ [33]. The Zeeman effect from the Helmholtz and nozzle-coil fields separates the 668.614 $\mathrm{nm}$ transition into six $+\sigma$, six $-\sigma$, and six $\pi$ subcomponents. Because of Doppler broadening, the six subcomponents of each merge into single peaks called the $+\sigma,-\sigma$, and $\pi$ components. To reduce the complexity of the LIF spectrum and increase signal/noise $(\mathrm{S} / \mathrm{N})$, the linearly polarized laser beam may be passed through a quarter-wave plate (QWP) oriented to convert the beam into either right- or left-circularly polarized
(RCP, LCP) light for exciting either the + or $-\sigma$ transitions. (For measurements of the perpendicular velocity, the laser beam is linearly polarized, parallel to the magnetic field.) The laser beam is modulated at $\sim 4 \mathrm{kHz}$ by a mechanical chopper, for lock-in detection of the $442.7 \mathrm{~nm}$ fluorescence. This LIF system operates well within the unsaturated mode wherein the LIF signal is proportional to the laser intensity and the $3 d^{4} F_{7 / 2}$ metastable population in the detection volume that is in resonance with the laser. A single wavelength scan is usually limited to a mode-hop-free region of $0.021 \mathrm{~nm}(14 \mathrm{GHz})$ and is performed in $\sim 60 \mathrm{~s} ; 1000$ data points are recorded. Simultaneous with each wavelength scan, laser power and wavelength are recorded using a ThorLabs powermeter and a Burleigh 1500 wavemeter (resolution $= \pm 0.0001 \mathrm{~nm}$ ), respectively. A set of observations of a single plasma condition typically includes scans over several adjacent wavelength regions in the range of $668.580-668.660 \mathrm{~nm}$, both with and without the QWP inserted. Using orthogonal QWP orientations allows the Zeeman splitting to be measured and the unshifted line center to be identified.

The primary diagnostic for emission spectroscopy is a $1 / 2 \mathrm{~m}$ J-A spectrometer, equipped with a $256 \times 1024$-pixel Princeton Instruments intensified charge-coupled device (iCCD). Three gratings are mounted in the spectrometer: 600, 1200, and 2400 $1 / \mathrm{mm}$. The spectrometer is fiber-optic coupled to view the plasma using the same collection optics as the LIF system. Electron temperatures are extracted from the data using neutral-helium line ratios interpreted with a collisional-radiative model [34]. Accordingly, for these measurements helium is added to the argon plasmas. 

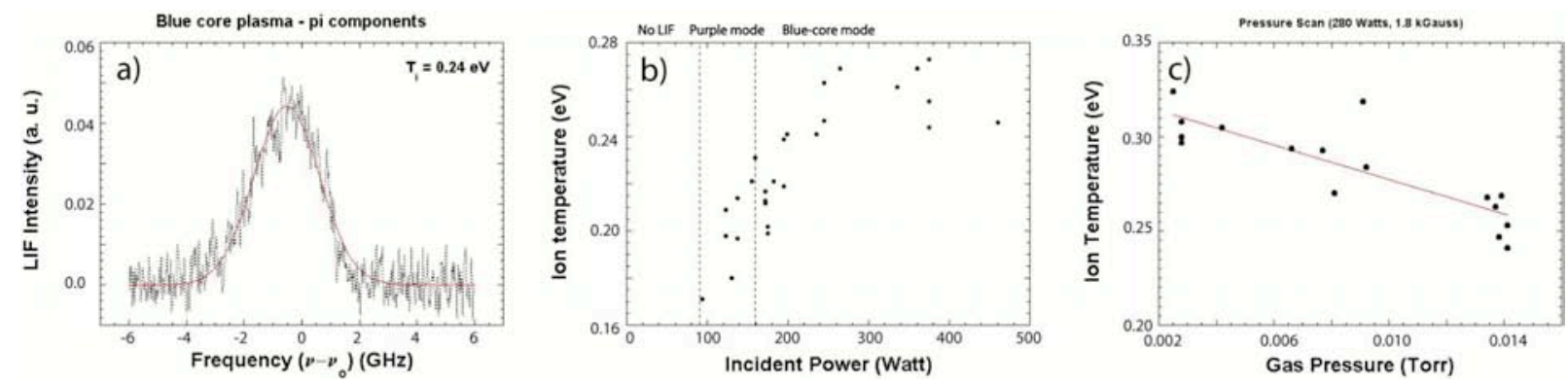

Fig. 2. (a) LIF intensity versus laser frequency, $\nu$, in the center of the MNX main chamber. $\nu_{o}$ is the frequency of the non-Doppler-shifted line. Uncertainty in the value of $\nu_{o}$ is $0.5 \mathrm{GHz}$. (b) $T_{i, \perp}$ versus RF power. At this argon fill pressure, the plasma makes a transition from inductive (purple) to helicon (blue-core) mode at powers above $160 \mathrm{~W}$. Though plasma exists at powers as low as $20 \mathrm{~W}$, the LIF signal is too weak to provide a good measurement of ion temperature. (c) $T_{i, \perp}$ versus argon fill pressure. (Color version available online at http://ieeexplore.ieee.org).
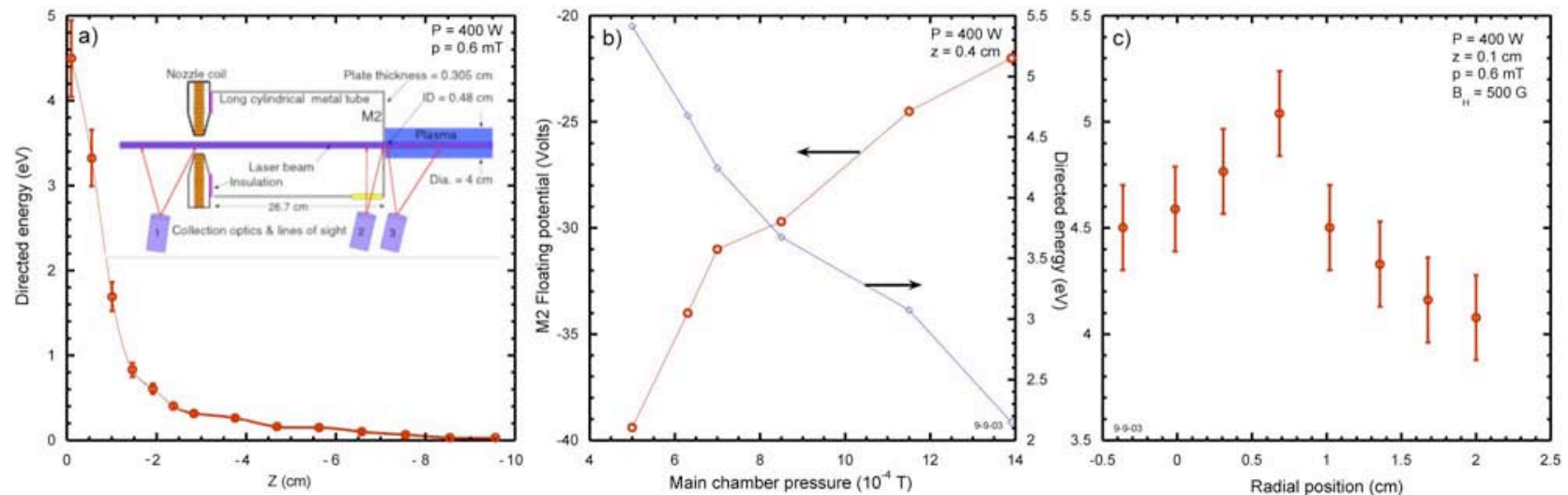

Fig. 3. Z-directed argon-ion energy, $E_{z}$, in the $\mathrm{MNX}$ main chamber for $P_{\mathrm{RF}}=400 \mathrm{~W}$. (a) $E_{z}$ near M2 versus $z$, using collection optics 3, as shown in the inset. Note that M2 was relocated to near the center of MNX's main chamber and the diameter of the aperture was set to $0.48 \mathrm{~cm}$. (b) Variation of $E_{z}$ (at $z=$ $0.1 \mathrm{~cm}$ ) versus main chamber pressure. Floating potential of M2 becomes more negative with decreasing pressure. (c) Variation of $E_{z}$ versus radial position. LIF collection optics viewed at the aperture as the plasma column was shifted upwards by addition of a vertical magnetic field. (Color version available online at http://ieeexplore.ieee.org).

\section{LIF-MEASURED ION POPULATIONS}

The $A r^{+*}$ perpendicular temperature, $T_{i, \perp}$, was measured on-axis in the center of the MNX main chamber using LIF on the $\pi$ component. Fig. 2(a) shows one LIF spectrum. A deconvolution technique [35] was used to extract the temperature and ion flow velocity. The ions are relatively cool and have negligible radial flow velocity. The red curve shows the best fit of a Gaussian to the data, giving $T_{i, \perp}=0.24 \mathrm{eV}$. Ion temperature was measured at a variety of RF power levels and Ar fill pressures, $p_{n}$. Fig. 2(b) shows $T_{i, \perp} v s$. RF power at a fill pressure of $5 \mathrm{mT}$. At low power the plasma is in the inductively coupled (so-called "purple") mode. The threshold for the transition to blue-core depends on the fill pressure, with lower powers being required at higher pressures. Fig. 2(c) shows that $T_{i, \perp}$ increases with decreasing $p_{n}$. At the lowest operating pressure where a stable helicon discharge could be sustained with this antenna system, $0.4 \mathrm{mT}, T_{i, \perp}$ reached $0.6 \mathrm{eV}$. As noted later, the plasma density in MNX's "blue-core" mode is about $10^{13} \mathrm{~cm}^{-3}$; these ions are highly collisional, with a Coulomb mean-free-path, $\lambda_{i i}$, less than $3 \mathrm{~mm}$.

The parallel ion-energy distributions versus axial position near the center of the MNX main chamber were measured along the axis of MNX, Fig. 3(a), using the $\sigma$ components [36]. To allow better lines-of-sight for the LIF optics, M2 was relocated to near the center of MNX by placing it on a long metal cylinder attached to the nozzle coil, see Fig. 3(a) inset. The data shown were obtained using collection optics 3 . At a position $7 \mathrm{~cm}$ from $\mathrm{M} 2$, the ions are cold, $T_{i, \|} \sim 0.2 \mathrm{eV}$, and unflowing. The ion flow velocity (energy) rises closer to M2, reaching $5 \mathrm{eV}, \sim T_{e}$, at $1 \mathrm{~mm}$ from M2. This region of ion acceleration is the presheath; its length, $\sim 3 \mathrm{~cm}$, is comparable to the ion-neutral charge-exchange mean-free-path, $\lambda_{i n}$. (In the plasma core, $\lambda_{i n}$ in Table I may be an underestimate because of the reduction in neutral density there.) The ion temperature, both parallel and perpendicular, is of order $0.1-1 \mathrm{eV}$, allowing the pre-sheath ions to equilibrate with each other.

The maximum $z$-directed energy $E_{z}$ attained by ions in the presheath decreases with increasing pressure, see Fig. 3(b). As shown in Section IV, the electron temperature in this low pressure range drops with increasing neutral pressure; the ion energy at the aperture remains $\sim T_{e}$. Also shown in Fig. 3(b) is the floating potential of $\mathrm{M} 2,{ }_{M 2} V_{f}$. At a power of $1 \mathrm{~kW}{ }_{M 2} V_{f}$ has fallen to about $-50 \mathrm{~V}$ at $p_{n}=0.6 \mathrm{mT}$.

The plasma column may be shifted vertically or horizontally by addition of fields provided by pairs of coils positioned outside the Helmholtz coils. Using this capability, the plasma column was moved vertically while $E_{z}$ was measured $1 \mathrm{~mm}$ from the aperture. The presheath $E_{z}$ radial profile is slightly hollow, see Fig. 3(c). ${ }_{M 2} V_{f}$ remained constant at $-32.1 \mathrm{~V}$.

We have explored the effect of M2 location on the energy of the downstream $A r^{+*}$ by placing M2 at four locations, three 

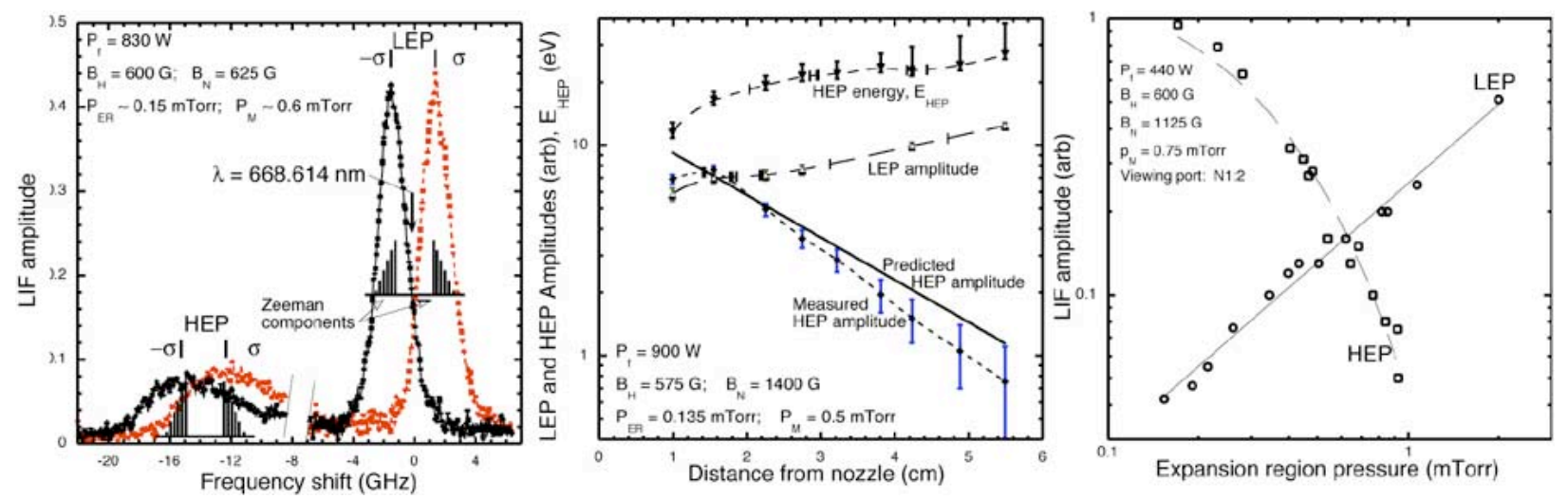

Fig. 4. (a) LIF amplitude versus frequency shift in the ER, $2 \mathrm{~cm}$ downstream from the nozzle midplane. Frequency zero is the non-Doppler-shifted line. Red (black) data are for RCP (LCP) laser beam. Six Zeeman + and $-\sigma$ subcomponents merge into single peaks. Two sets of peaks are labeled, HEP and LEP. (b) HEP amplitude and $E_{z}$ and LEP amplitude versus distance downstream from the nozzle coil. (c) HEP and LEP amplitudes versus argon pressure in the ER at $2 \mathrm{~cm}$ from the nozzle-coil midplane.

at various distances $(0.1,1.9$ and $26.7 \mathrm{~cm})$ upstream of the magnetic nozzle and one $3 \mathrm{~cm}$ downstream of the nozzle [36]. We have also removed M2 completely, allowing the nozzle coil, either through its magnetic field or its 2-cm inner diameter, to act as an aperture for the 4-cm-diameter Ar plasmas. The results for these five cases are qualitatively the same, though important differences occur. Herein, we review the primary common feature, the acceleration of ions to supersonic speed. In conjunction with a sharp decrease in density downstream of M2, these higher energy ions are collisionless.

Fig. 4(a) shows the LIF spectral distributions for both RCP and LCP laser beams. Two $+\sigma$ and two $-\sigma$ peaks are seen. The + /- pair with little frequency shift are termed the low energy peaks (LEP); the Doppler shift of their average frequency shift is subthermal. The $+/-$ pair with large frequency shift are termed the high energy peaks (HEP). The parallel temperatures of the LEP and HEP ions are about $0.1 \mathrm{eV}$ and $1 \mathrm{eV}$, respectively. $E_{z}$ of the HEP increases with distance downstream of the nozzle coil, see Fig. 4(b). Energies over $70 \mathrm{eV}$ have been observed, depending on the placement of M2, the RF power, and the gas pressure in both the main chamber and the ER. (Efforts to reach the $180 \mathrm{ev}$ target, i.e., an $I_{s p}$ of $3000 \mathrm{~s}$, are being made.) Increasing gas pressure in the ER decreases the amplitude of the HEP signal while raising that of the LEP, see Fig. 4(c). With M2 placed in different locations for better LIF access, the length of the region of acceleration to an energy above $2 T_{e}$ has been measured to be $0.3-0.6 \mathrm{~cm}$.

The LEP ions in the ER are created by electron impact ionization [16] and possibly CX. In contrast, the HEP ions were created in the main chamber(ascoldions) and accelerated through a double layer formed just down stream of M2 or the nozzle coil. The decrease in HEP amplitude in the ER, see Fig. 4(b) is slightly faster than predicted by a model which includes magnetic-field divergence, ion acceleration, and de-excitation of the $A r^{+*}$ 's by collisions with neutrals. The mean-free-path of the HEP ions against charge exchange is as long as $30 \mathrm{~cm}$ and against Coulomb collisions with the background plasma is greater than several meters while the LEP ions remain collisional until the density falls below $10^{10} \mathrm{~cm}^{-3}$. The ER plasma thus consists of both collisionless and collisional ions populations, the former flowing through the latter at supersonic speed.
One goal of the MNX program is to explore the analogy between a magnetic nozzle and a Laval nozzle. Fluid-even multicomponent-upstream of a Laval nozzle is generally in thermal equilibrium. The perpendicular component of the fluid's thermal energy gets converted by the nozzle to directed energy. In the MNX main chamber, collisional coupling is weak between electrons and ions and there is no thermal equilibration between them; the ions are much cooler than the electrons. The gain in energy by the ions as they transverse the nozzle is far more than the conversion of their own perpendicular energy to parallel energy. They are gaining energy from the electrons, with a sizable fraction of the energy gained in a region short, $c a .100 \lambda_{D}$, compared to the nozzle field curvature. From measurements described in Section IV, we shall see that the energy gained by ions is greater than that available from the thermal electrons. To elucidate the energy-gain process, we present here effects of nozzle field strength on the ion energy.

Fig. (5) shows the $E_{z}$ for ions downstream of M2 versus nozzle field strength $B_{n}$ for three locations of M2. For M2 placed immediately upstream of the nozzle coil, see Fig. 5(a) inset, the directed ion energy of the HEP ions first falls $10 \%$ with increasing $B_{n}$ to $500 \mathrm{G}$ then slowly rises $5 \%$ as $B_{n}$ reaches its maximum value. Fig. 5(b), with M2 placed $3.1 \mathrm{~cm}$ downstream of the nozzle coil shows much more dramatic reduction in the $E_{z}$ of the HEP ions-by more than $50 \%$ whether $1 \mathrm{~mm}$ from the M2 aperture, $z=3.2 \mathrm{~cm}$, or $0.8 \mathrm{~cm}$ further downstream, at $z=4.0 \mathrm{~cm}$. Fig. $5(\mathrm{~b})$ also shows that the brightness of the HEP increases with $B_{n}$, an indicator that more ion flux is channelled through the nozzle coil with increase in $B_{n}$, i.e., $B / n_{e} \sim$ constant.

The data in Fig. 5(c) require a bit more explanation. With M2 placed well upstream of the nozzle coil, at $z=-26.7 \mathrm{~cm}$, three sets of peaks are observed in the LIF spectrum in the ER. The HEP and LEP have already been discussed. Appearing at an intermediate energy is a third set of peaks called the MEP, medium energy peaks [36]. These are ascribed to plasma formed within the long metal cylinder. The energy of these MEP ions grows $50 \%$ as $B_{n}$ increases to $2.5 \mathrm{kG}$. The MEP amplitude grows faster than $\propto B_{n}$.

Changing $B_{n}$ has little effect on the ion temperature and flow in the main chamber. In the next section, we show that $B_{n}$ also 

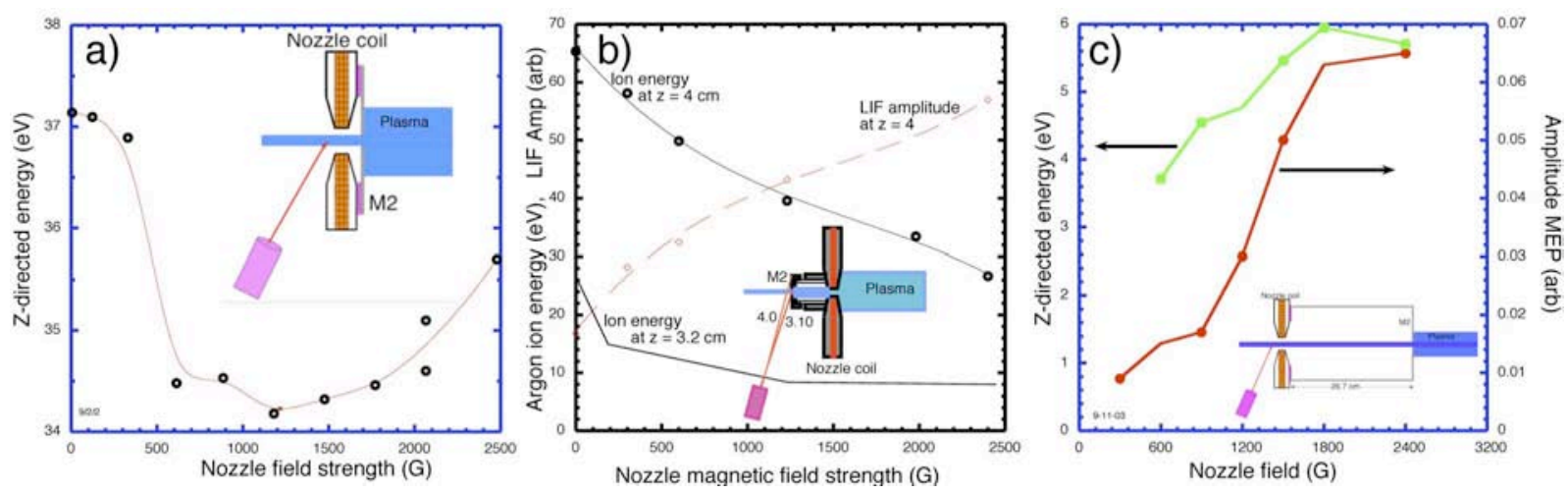

Fig. 5. (a) $E_{z}$ of the HEP ions versus nozzle-field strength at $z=2 \mathrm{~cm}$ in the ER. Plate M2 was located $1 \mathrm{~cm}$ upstream of the nozzle coil midplane, as shown in the inset. (b) HEP $E_{z}$ versus nozzle-field strength at $\mathrm{z}=3.2$ and $4.0 \mathrm{~cm}$ from the nozzle-coil midplane. M2 was located $3.1 \mathrm{~cm}$ downstream of the nozzle-coil midplane, see inset. (c) $E_{z}$ of the MEP ions versus nozzle field strength at $\mathrm{z}=2 \mathrm{~cm}$ from the nozzle-coil midplane. M2 was located $26.7 \mathrm{~cm}$ upstream of the nozzle-coil midplane, see inset. (Color version available online at http://ieeexplore.ieee.org).

has little effect on the temperature and density of the "bulk" electrons in these plasmas.

\section{Electric-Probe-MeAsured EleCtron Populations}

The probe data to be presented show that the ratio of the probe radius $r_{p}$ to the Debye length $\lambda_{D}$, is large, $c a$. 100, in both the main chamber and the ER. That fact, combined with the LIF observations that no plasma drift occurs in the main chamber (except in the presheath), allows the Laframboise equations for cylindrical probes to be used in the main chamber to evaluate the Langmuir probe data. An important feature of the Laframboise equations for large $r_{p} / \lambda_{D}$ is a flat ion-saturation current with increasingly negative bias. The main-chamber Langmuir-probe characteristics often show a small tail extending to high energy, above $100 \mathrm{eV}$. We do not discuss that data here, due to the lack of certainty that RF is not affecting the characteristics. Instead we describe the bulk electron parameters in the main chamber and then the tail in the ER probe characteristic where RF effects are unimportant.

The bulk electron populations may be characterized by temperature, density, and ion saturation current. How these depend on magnetic field, main-chamber neutral pressure $\left(p_{n}\right)$, and RF power $\left(P_{\mathrm{RF}}\right)$ are shown in Fig. 6. All data are from blue-coremode helicon discharges. Fig. 6 consists of eight graphs in two columns. Graphs in the left column are of ion saturation current versus radius with the probe biased at $-60 \mathrm{~V}$. Ion saturation current $I_{\text {sat }}$ is plotted instead of density because it is acquired with better spatial resolution. Also, the electron temperature is nearly constant across the radius, so $I_{\text {sat }}$ gives a good relative measure of $n_{e}(r)$. The graphs in the right column are of the central values of plasma density and electron temperature versus the independent variables: main chamber pressure, a) and b); RF-power coupled, c) and d); nozzle-coil current, e) and f); or Helmholtz-(main)-coil current, g) and h). In all cases, the $I_{\text {sat }}$ radial profiles are peaked. Considerable plasma exists beyond $r=2 \mathrm{~cm}$, the nominal plasma edge defined by the inner diameter of the Pyrex section inside the helicon antenna.

The data for Fig. 6(a) and (b) were acquired at a $P_{\mathrm{RF}}=$ $800 \mathrm{~W}, B_{n}=0 \mathrm{G}$, and $B_{H}=500 \mathrm{G}$. The electron temperature is highest at low $p_{n}$. This is consistent with the model of plasma formation based on a balance between sheath-limited particle and energy losses and volumetric ionization [5]. The density is low at low and high $p_{n}$. The data for Fig. 6(c) and (d) were acquired at $p_{n}=2.5$ and $0.7 \mathrm{mT}, B_{n}=0 \mathrm{G}$, and $B_{H}=500 \mathrm{G}$. The electron temperature is nearly independent of $P_{\mathrm{RF}}$ while the density rises monotonically with $P_{\mathrm{RF}}$, again consistent with [5]. The data for Fig. 6(e) and (f) were acquired at a $P_{\mathrm{RF}}=800 \mathrm{~W}$, $p_{n}=0.7 \mathrm{mT}$, and $B_{H}=500 \mathrm{G}$. The electron temperature falls slightly with $B_{n}$ while the density rises. Energy confinement remains constant. The data for Fig. $6(\mathrm{~g})$ and $(\mathrm{h})$ were acquired at a $P_{\mathrm{RF}}=800 \mathrm{~W}, p_{n}=0.7 \mathrm{mT}$, and $B_{n}=0 \mathrm{G}$. The electron temperature depends little on Helmholtz-coil current, $I_{H}$. The density falls precipitously for $I_{H}<80 \mathrm{~A}$. We are unable to sustain helicon discharges at $I_{H}<37 \mathrm{~A}$.

Langmuir-probe characteristics obtained with a paddle probe located in the ER at $z=12 \mathrm{~cm}$ from the nozzle coil are shown in Fig. 7 for Ar plasmas. A constant ion-saturation current was subtracted from the probe current. The operating parameters were nearly the same for both (a) and (b), with the exception that the nozzle coil was used while taking the data in Fig. 7(b). Without the nozzle coil powered, a clear two-temperature Maxwellian is seen; with the nozzle coil powered, the separation in temperatures in less pronounced and the bulk electrons are warmer. Recall that $E_{z}$ in the ER dropped with increasing $B_{n}$. The tail temperature is strongly dependent on the exact value of the saturation current subtracted. If a higher ion-saturation current is subtracted, the tail loses its Maxwellian shape, i.e., exponential decrease with $V_{\text {bias }}$, and a higher average energy is inferred.

We now describe electrical measurements on macroscopic structures in MNX and how they indicate the presence of fast electrons in the main chamber. Fig. 8(a) adds M2 (apertureplate) floating potential, ${ }_{M 2} V_{f}$, and the ER pressure, $p_{\mathrm{ER}}$, results to data previously presented on the HEP ion energy, $E_{z}$, in the ER with the M2 aperture plate positioned at $3.1 \mathrm{~cm}$ in the ER, see inset Fig. 5(b). The pressure in the ER increases with $B_{n}$, indicating increasing plasma flow through the M2 aperture. $\left|{ }_{M 2} V_{f}\right|$ and $E_{z}$ both decrease with increasing $B_{n}$. Note that ${ }_{M 2} V_{f} \sim 10 T_{e}$ in the main chamber, about twice the value expected based on the data in Fig. 6. This is a signature of a superthermal electron component in the main chamber [25], [27], [37], [38]. In simple terms, when the fast electron flux is comparable to the ion flux, the bulk electrons are not needed to balance 

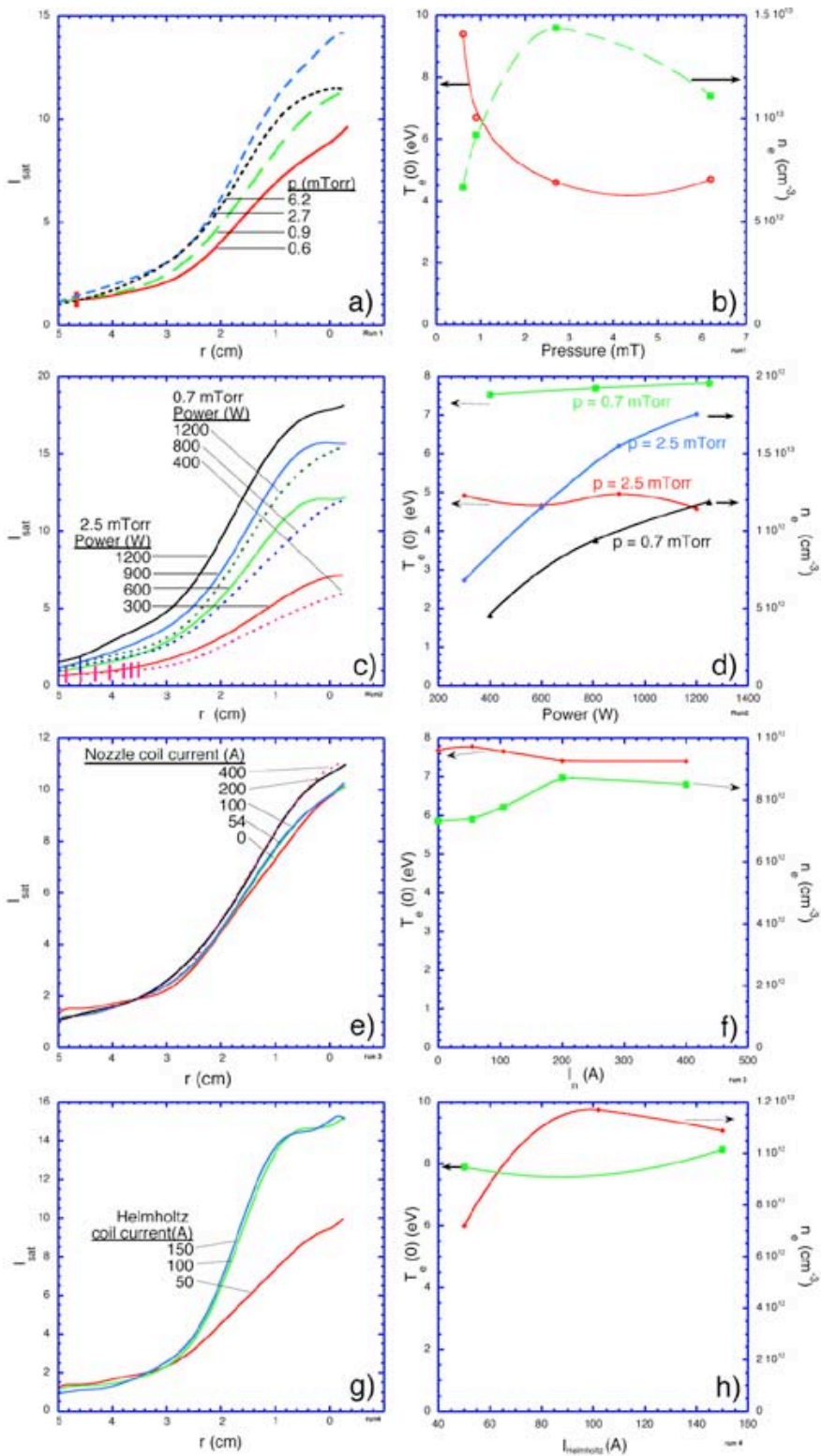

Fig. 6. Radial dependance of ion saturation current and dependences of central electron temperature and density of bulk plasma in center of MNX main chamber versus main chamber pressure, RF power, nozzle-coil current, and Helmholtz-coil current.

the ambipolar flow to objects immersed in the plasma; the objects will charge to a higher floating potential, approaching the energy of the fast electrons. For argon plasmas this would occur at a fast electron density above $\sqrt{m_{e} / M_{i}} n_{e} \sim 0.003 n_{e}$ [37], [38].

An immediate question is whether the M2 potential sets the ion energy or is result of the same phenomenon that generates high energy ions. Fig. 8(b) shows that the M2 potential is not the cause of ion acceleration. The ion energy measured at $z=$ $3.2 \mathrm{~cm}$ barely changes as the voltage applied to M2 - using the main-chamber stainless-steel vessel as ground-is varied both above and below the floating potential, $-40 \mathrm{~V}$. The constancy of $p_{\mathrm{ER}}$ shows that the aperture plate voltage barely affects the plasma flow into the ER. Fig. 8(b) also shows that the presheath potential, as determined from $E_{z}$ in the aperture, $c a .8 \mathrm{eV}$, is hardly affected by biasing M2.
In the ER are placed 15 copper rings-also called flux conservers-coaxial with the MNX major axis and separated from each other by $1.1-1.3 \mathrm{~cm}$. Eight of the rings are electrically biasable and instrumented to measure currents or floating potentials, see Fig. 9(a). Floating potentials ${ }_{r} V_{f}$ of the rings were measured with M2 placed either in the ER or in the MC. Fig. 9(b) shows ${ }_{r} V_{f}$ for both M2 locations and at similar MNX operational parameters. The first feature seen from these measurements is an increasingly negative floating potential with increasing distance from the nozzle, until ring number 6 or 7 . (Aperture plate M3 was typically several volts more positive than ring number 8.) The ${ }_{r} V_{f}$ with the ER M2 is much lower than the MC M2. Recall that the HEP ions reach a much higher energy, $E_{z} \sim 70 \mathrm{eV}$, with the ER M2 than the MC M2, $E_{z} \sim$ $30 \mathrm{eV}$. Grounding M2 makes little difference in ${ }_{r} V_{f}$. We attribute the large negative ${ }_{r} V_{f}$ to an energetic electron population in the ER. When $B_{n}$ was increased, the amplitude of ${ }_{r} V_{f}$ fell by about a factor of 2 at full $B_{n}$ field. An explanation for the effect on $V_{f}$ is that the fast electron population has a high pitch angle, i.e., $v_{\perp}>v_{\|}$. Note that the early explanation for hot electrons in helicon plasmas, Landau damping, generates high $v_{\|}$[39].

Fig. 9(c) shows the effect on current to the rings with the rings biased at $-1 \mathrm{~V}$. The exponential decrease of current with distance is consistent with a linear decrease in floating potential, if the fast electron distribution is Maxwellian. The currents measured with a high nozzle field, though initially higher than with $B_{n}=0$, decrease faster with distance, indicating a less energetic electron population in the ER with the nozzle field.

\section{Spectroscopic Measurements of Electron EnERGy}

Using a 1/2-m J-A spectrometer equipped with an iCCD, spectra in the visible wavelength range were measured in the main chamber. Fig. 10(a) shows the Abel-inverted brightness radial profiles of two lines, one for Ar I $(763.5 \mathrm{~nm})$ and the other for Ar II (434.8 nm). These measurements, made in the blue-core mode, show whence the blue core gets its name, from the strongly peaked bight blue of the argon ion lines. Fig. 10(b) shows the ratio of brightnesses, $\left(B_{A r I}\right)^{2} / B_{A r I I}$, where $B_{i}$ stand for the brightness of the $i$ th line. If collisional excitation were responsible for the emission, then $B_{A r I}=n_{e} n_{A r I}\langle\sigma v\rangle_{I}$, $B_{A r I I}=n_{e} n_{A r I I}\langle\sigma v\rangle_{I I}$, and the ratio would be constant if the argon neutral density $n_{A r I}$ and the electron-energy-distribution shape were independent of radius. The ratio is not constant, which could be explained by burnout of $n_{A r I}$ on axis-estimated to be likely at the high densities in MNX - or if the relative population of the fast electrons changes with radius, for which we have counter evidence, see Fig. 8(b). Burnout could explain the flattening of $p_{\text {ER }}$ seen in Fig. 8(a) with increasing $B_{n}$.

If the plasma density is known, measurements of He I line ratios and use of a collisional-radiative model, such as that of Sasaki et al. [34], should allow the electron temperature to be calculated. A comparison of a "corrected" Sasaki model with experiment [40] showed very poor agreement at low density, $n_{e}<10^{11} \mathrm{~cm}^{-3}$, but improving agreement as the density approached $10^{12} \mathrm{~cm}^{-3}$. At $T_{e}=10 \mathrm{eV}$ and $n_{e}=10^{12} \mathrm{~cm}^{-3}$, the experimentally measured line ratios exceeded those expected based on the probe measurements by $20 \%$ [40], corresponding 

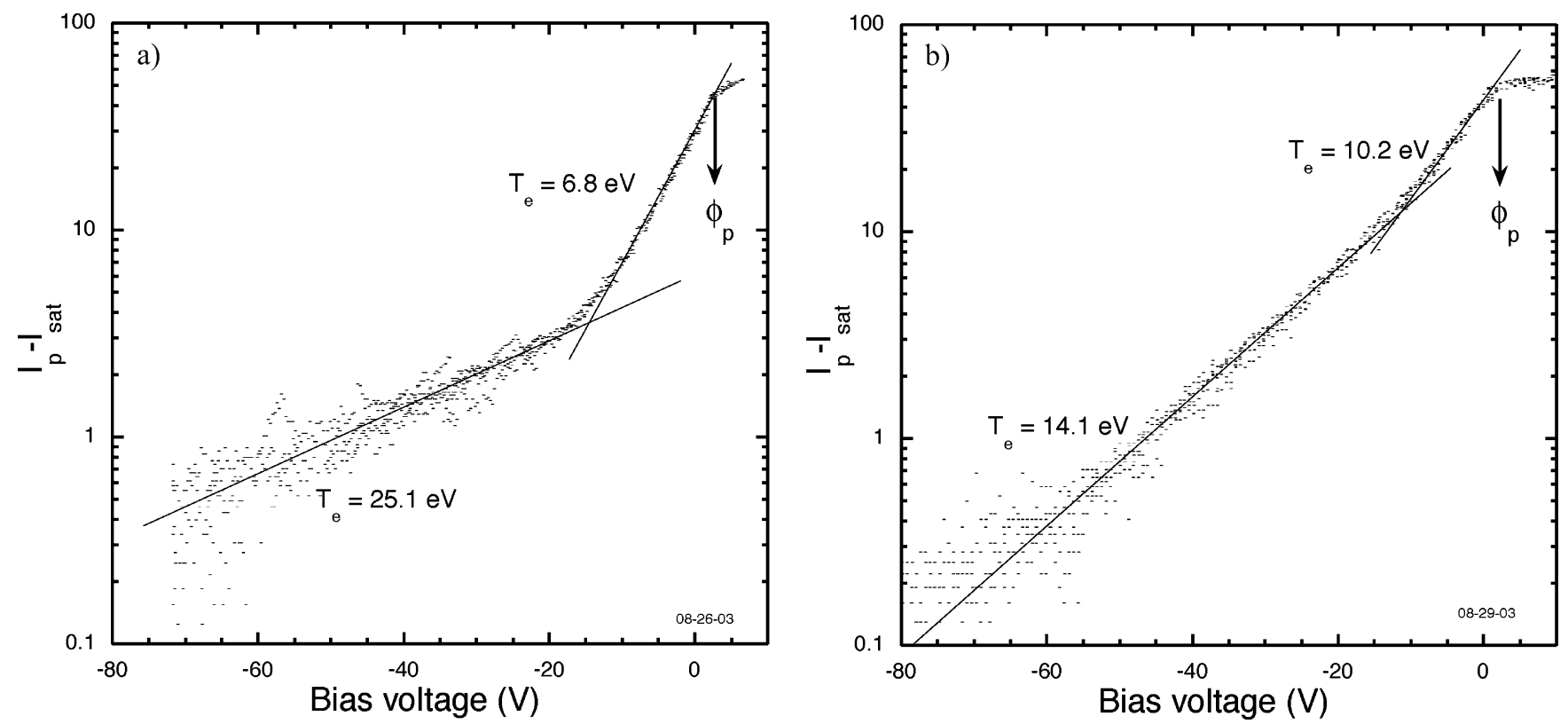

Fig. 7. Langmuir-probe characteristics in the ER. $P_{\mathrm{RF}} \sim 900 \mathrm{~W}, p_{n} \sim 0.7 \mathrm{mT}, B_{H} \sim 700 \mathrm{G}$. (a) No nozzle field. (b) With 2-kG nozzle field. Error bars: $\pm 3 \%$ for bulk $T_{e} ; \pm 20 \%$ for tail $T_{e}$.
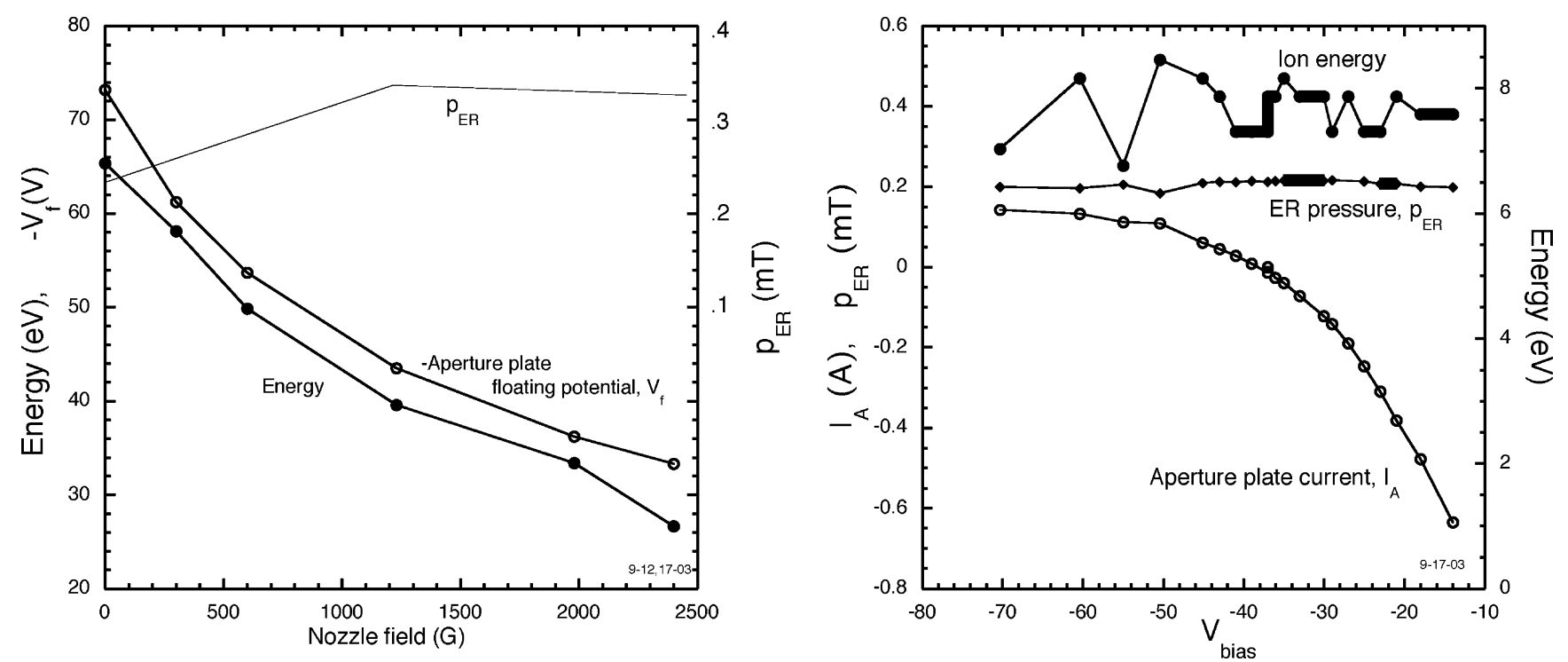

Fig. 8. (a) Dependence on nozzle-field strength of ER pressure, M2 floating potential and HEP $E_{z}$ at z $=4 \mathrm{~cm}$ in the ER. (b) ER pressure, HEP $E_{z}$ (at Z $=3.2 \mathrm{~cm}$ ) and M2 (aperture-plate) current versus bias voltage applied to M2. For geometry, see inset Fig. 5(b).

a temperature $\sim 10 \%$ higher. We have confirmed the poor fit in the low density range but also found cases of good agreement for pure helium discharges at considerably higher density, $1-5 \times 10^{13} \mathrm{~cm}^{-3}$, as predicted by the trend shown in Fig. 6 of [40]. Herein we present only select high-density cases which show great disagreements between probe measurements of $T_{e}$ and line-ratio measurements. In our experiments, probe measurements provided $n_{e}(r)$. As noted by [40], inclusion of a density profile improves the fit of the Sasaki model to the data.

The results of radial profile measurements are shown in Fig. 11(a) in which we have used the original Sasaki model interpreted with our probe-measured central density. For Fig. 11(a), the Sasaki method gives a twice higher electron temperature than the probes. Note that our use of the central density underestimates the temperature inferred from the line-ratio model, hence the factor of 2 discrepancy is a lower limit. The discrepancy is even more pronounced under other operating conditions. Fig. 11(b) compares the electron temperature using two lines ratios, $492.2 \mathrm{~nm} / 471.3 \mathrm{~nm}$ and $504.8 \mathrm{~nm} / 471.3 \mathrm{~nm}$, with that obtained by the Langmuir probe. These discharges were prepared by starting with a pure helium helicon plasma, for which probes show a twice higher electron temperature than pure argon plasmas. To these plasma we added increasing flows of argon. The discharges were not in the blue-core mode. The Sasaki method is particularly susceptible to populations of fast electrons. The high temperatures indicated by the Sasaki method could be explained by a small population of 100-200 $\mathrm{eV}$ electrons added to a $5 \mathrm{eV}$ Maxwellian. Collisions of $\mathrm{He}$ with Ar, not included in the Sasaki model, may also strongly affect the line ratios. 
a)
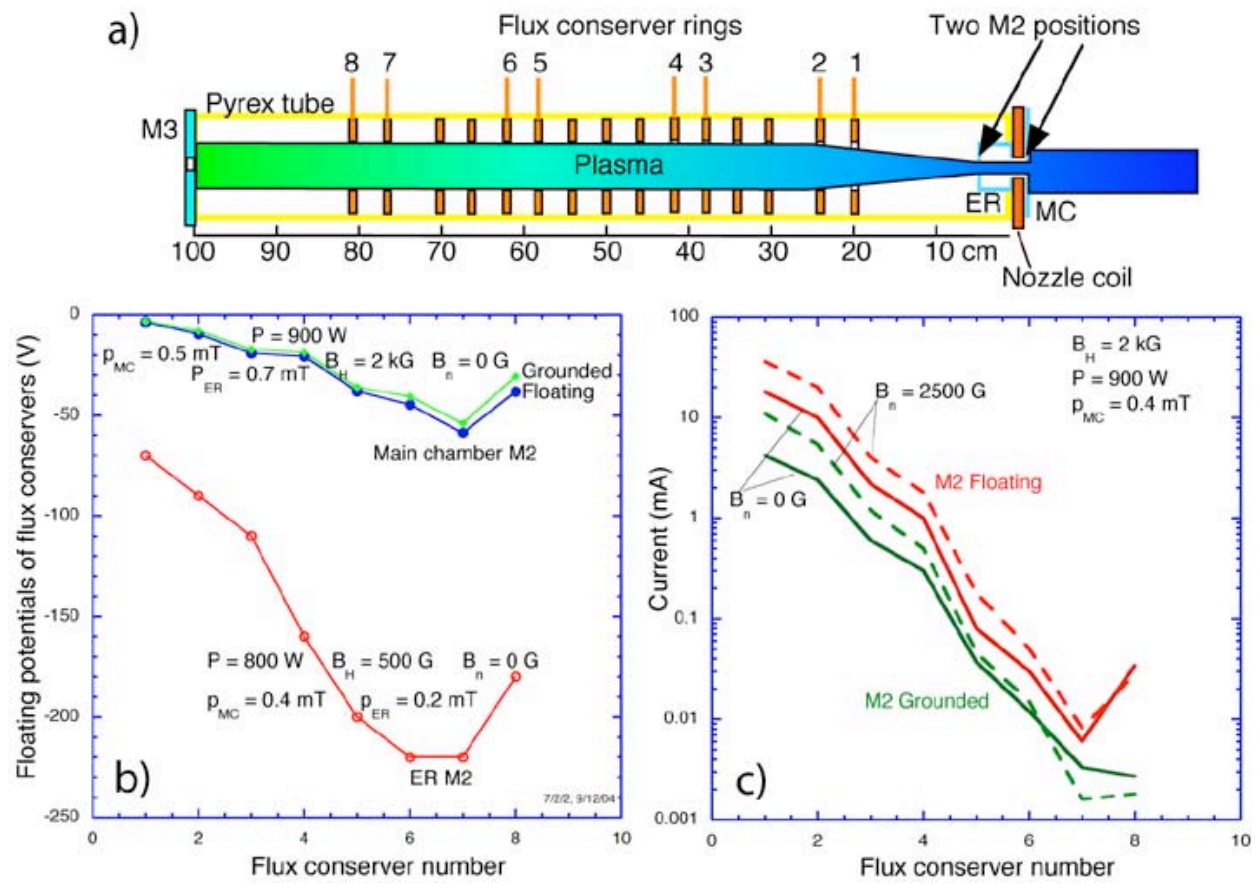

Fig. 9. (a) Schematic showing locations of 8 instrumented flux-conserver (copper) rings and the two positions of the M2 aperture plate used in this series of experiments. (b) Floating potentials of 8 instrumented flux-conserver rings for two different M2 positions. Nozzle field was zero. Adding a nozzle field approximately halves the amplitude of the floating potentials. (c) Current drawn by flux-conserver rings with and without the nozzle coil powered and with and without M2 grounded. MC M2 position was used.
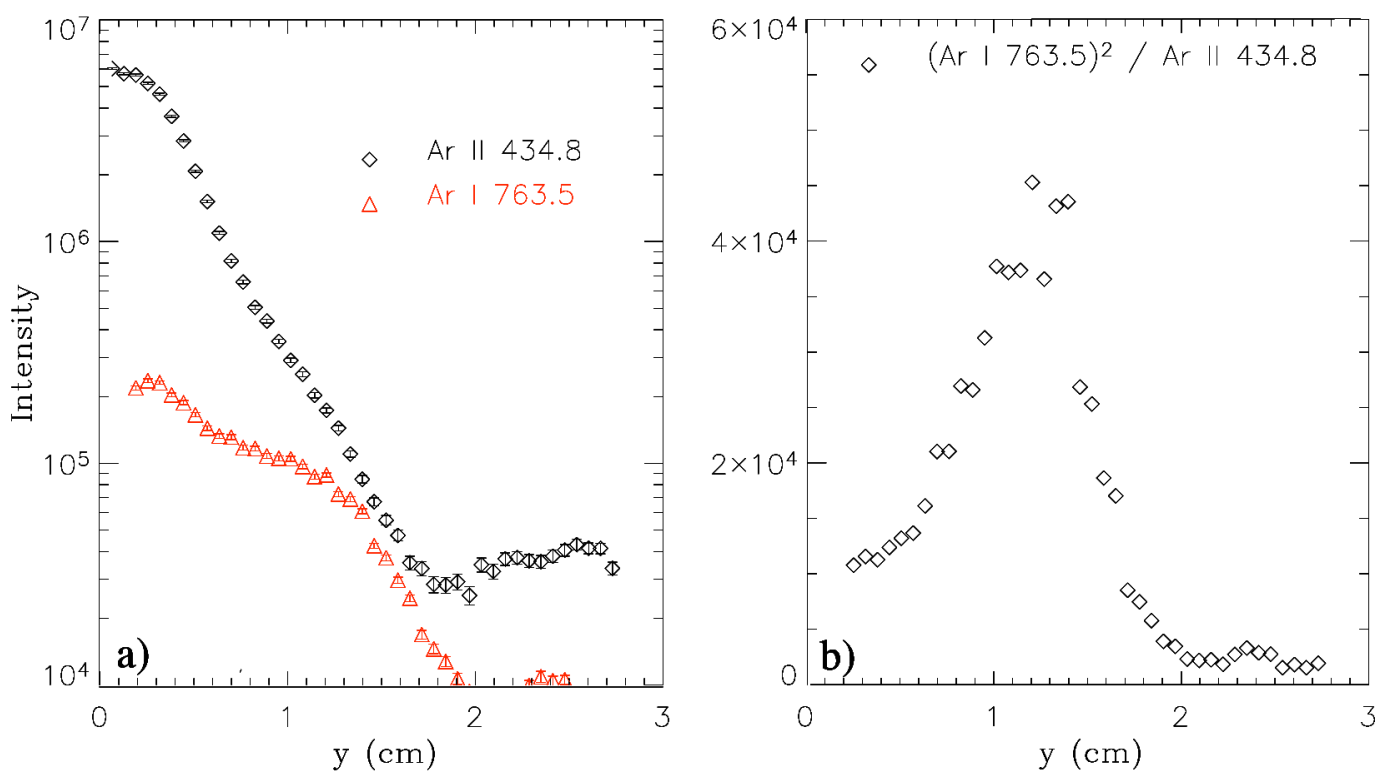

Fig. 10. (a) Abel-inverted brightnesses of an Ar I and an Ar II spectral line. (b) Ratio of brightnesses, $\left(B_{A r I}\right)^{2} / B_{A r I I}$, as a function of radius. (Color version available online at http://ieeexplore.ieee.org).

\section{SUMMARY AND FUtURE WORK}

The LIF measurements show that a low temperature, high density — hence highly collisional—ion population exists in the MNX main chamber. A presheath accelerates these ions to above the sound speed, $E_{z} \sim T_{e}$, near apertures. This higher-than-expected energy is suggested to be the result of a superthermal electron population of energy $10 T_{e}$ and density less than $0.003 n_{e}$ in the main chamber [36]. Superthermal electron beams have been found in helicon plasmas by a number of research teams [41]-[43]. If the superthermal electrons were responsible for accelerating the ions to superthermal speeds, the power transfer would be effected in the DL region and amount to $2 n_{i} c_{s} q_{i} V_{D L}$ evaluated at the DL presheath boundary, $\approx 10 \mathrm{~W} / \mathrm{cm}^{2}$. Whether the superthermal electrons are produced by Landau damping or by acceleration upstream through the double layer or by another mechanism is not resolved. The ions in the $\sim 3$-cm-long presheath retain their low parallel and perpendicular temperatures, $c a .0 .1-1 \mathrm{eV}$, hence remain highly collisional with each other. After passing through apertures, whether mechanical or magnetic, the ions are accelerated to superthermal speeds by a double layer of strength $\Delta \phi_{D L} \sim 3-10 T_{e}$, the higher value suggested to be the result of a tenuous superthermal electron population in the 

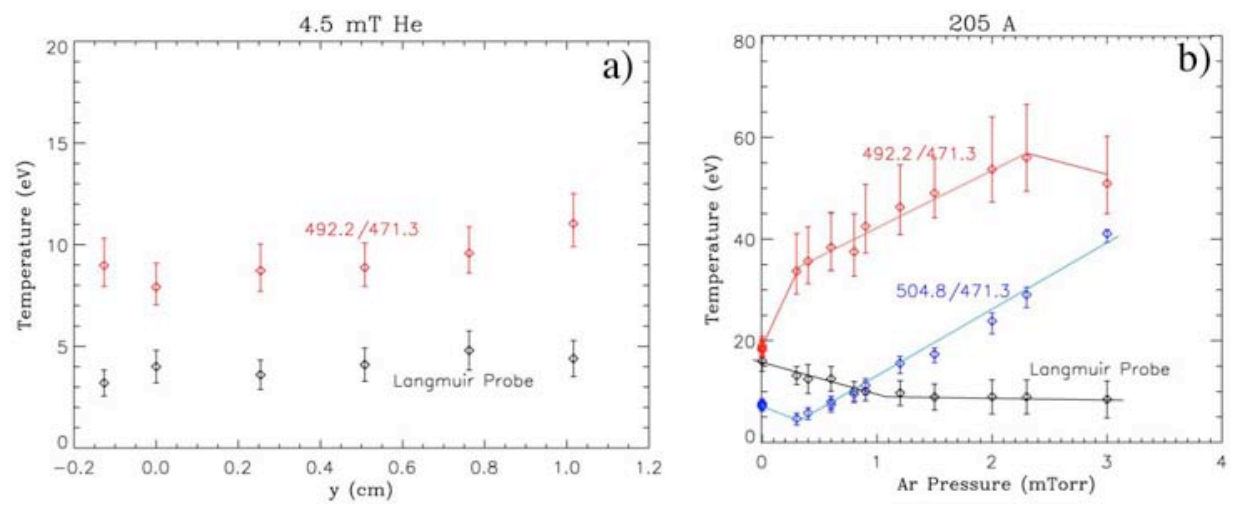

Fig. 11. (a) Radial profiles of $T_{e}$ determined by the Sasaki et al., [34] collisional-radiative model and with the Langmuir probe. (b) Dependence of three calculated electron temperatures, using probe data and the Sasaki method, on argon fill pressure in a helium helicon discharge. (Color version available online at http:// ieeexplore.ieee.org).

main chamber. The same attribution has been made for strong double layers found in lower density plasmas [25], [27]. As these superthermal ions flow through the expansion region (ER) they may interact with a cold $\left(T_{i} \sim 0.2 \mathrm{eV}\right)$, stagnant plasma, formed by local ionization, or with the neutrals. Future research will examine whether such interactions lead to streaming instabilities.

Langmuir-probe characteristics in the ER (and main chamber) show a non-flat ion saturation current, extending beyond a bias voltage of $-140 \mathrm{~V}$. This may be attributable to a superthermal tail. The bulk electron temperature in the main chamber is typically $2-8 \mathrm{eV}$ for Argon plasmas. Pure helium plasmas have a higher bulk temperature.

Floating potentials of macroscopic metal structures located in the main chamber and in the ER give convincing evidence for superthermal electrons. In particular, a series of copper rings in the ER reach floating potentials as great as $-220 \mathrm{~V}$. That the floating potentials become less negative with increasing nozzle field strength might occur if the fast electron population has a high pitch angle. The mechanism for creating a high pitch-angle distribution has not been found. The superthermal electrons are collisionless, as shown by the great distance in the ER at which the large negative floating potentials occur. Probe measurements in the ER show a two-temperature electron distribution which is affected by the nozzle field.

One of the research goals of MNX is to explore the trajectories of ions as they are accelerated to speeds exceeding the Alfvén speed. The main question is whether the ions will stay on the field lines or detach. Theoretical work predicts detachment when the flow speed exceeds the Alfvén speed [17]. Fig. 12 shows the measured axial ion speed as a function of axial position for the same aperture arrangement, as shown in Fig. 5(b). The nozzle coil is located at $z=0 \mathrm{~cm}$. Measurements of ion speed stopped about $10 \mathrm{~cm}$ from the nozzle due to low LIF signal strength. At the furthest point from the nozzle where there was good LIF signal-to-noise, the ion energy was about $75 \mathrm{eV}$. In these experiments the floating potential of the copper rings reached -220 volts. Based on this we extrapolate the ion velocity for both $\mathrm{Ar}^{+}$and $\mathrm{Ar}^{+2}$.

Also shown in Fig. 12 are the axial field strength and the local Alfvén speed, calculated under the assumption that the plasma

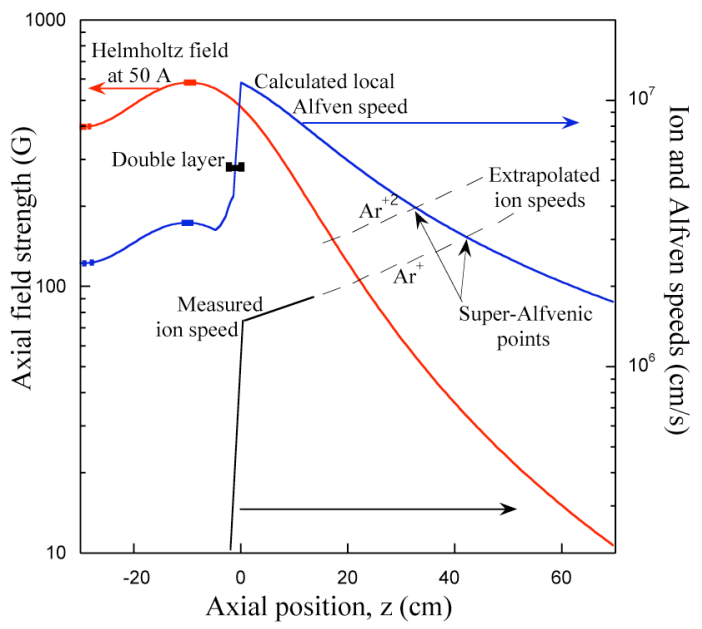

Fig. 12. Axial field strength (red), measured flow speed (black solid line), calculated Alfvén speed (blue) and extrapolated flow speeds (dashed black lines) for both $\mathrm{Ar}^{+}$and $\mathrm{Ar}^{+2}$ are shown. (Color version available online at http://ieeexplore.ieee.org).

density decreases with distance proportional to $B / v$, where $v$ is the ion flow speed and $B$ the magnetic field strength. The large jump in the Alfvén speed immediately downstream of the nozzle is due to the steep drop in plasma density at the double layer. Fig. 12 shows that the ion speed is expected to reach the Alfvén speed about $40 \mathrm{~cm}$ from the nozzle coil for $\mathrm{Ar}^{+}$and $30 \mathrm{~cm}$ for $\mathrm{Ar}^{+2}$. Studies of super-Alvénic ion flows in MNX will require reduction in background gas pressure and increases in LIF detection efficiency, to compensate for the diminished signal at increasing distance from the nozzle. Other technical challenges will have to be addressed to perform these daunting experiments.

\section{ACKNOWLEDGMENT}

The authors thank B. Berlinger for excellent technical support and Dr. Y. Raitses for useful conversations.

\section{REFERENCES}

[1] H. A. Blevin and P. J. Christiansen, "Propagation of helicon waves in a non-uniform plasma," Aust. J. Phys., vol. 19, p. 501, 1966.

[2] G. A. Baraff and S. J. Buchsbaum, "Surface-wave instability in heliconwave propagation," Phys. Rev., vol. 144, p. 226, 1966.

[3] R. W. Boswell and F. F. Chen, "Helicons-the early years," IEEE Trans. Plasma Sci., vol. 25, no. 6, pp. 1229-1244, Dec. 1989. 
[4] F. F. Chen and R. W. Boswell, "Helicons-the past decade," IEEE Trans. Plasma Sci., vol. 25, no. 6, pp. 1245-1257, Dec. 1989.

[5] M. A. Lieberman and A. J. Lichtenberg, Principles of Plasma Discharges and Materials Processing. New York: Wiley, 1994.

[6] A. J. Perry, D. Vender, and R. W. Boswell, "The application of the helicon source to plasma processing," J. Vac. Sci. Technol. B, vol. 9, p. 310, 1991.

[7] B. P. Cluggish, F. A. Anderegg, R. L. Freeman, J. Gilleland, T. J. Hilsabeck, R. C. Isler, W. D. Lee, A. A. Litvak, R. L. Miller, T. Ohkawa, S. Putvinski, K. R. Umstadter, and D. L. Winslow, "Density profile control in a large diameter helicon plasma," Phys. Plasmas, vol. 12 , p. 57101,2005

[8] R. W. Boswell, "Plasma production using a standing helicon wave," Phys. Lett., vol. 33A, p. 457, 1970.

[9] A. Komori, T. Shoji, M. Miyamoto, J. Kawai, and Y. Kawai, "Helicon waves and efficient plasma production," Phys. Fluids B, vol. 3, p. 893, 1991.

[10] F. F. Chen, "Plasma ionization by helicon waves," Plasma Physi. Controll. Fusion, vol. 33, p. 339, 1991

[11] F. Chang-Diaz, "The VASIMR rocket," Sci. Amer., vol. 283, p. 90, 2000

[12] J. A. Gilleland, "Application of a helicon discharge to electric propulsion," AIAA, vol. 98, p. 3934, 1998.

[13] R. H. Frisbee, "Advanced plasma propulsion for the 21st century," $J$. Propulsion Power, vol. 19, p. 1129, 2003.

[14] R. G. Jahn and E. Y. Choueiri, Electric Propulsion, in Encyclopedia of Physical Science and Technology, 3rd ed. New York: Academic, 2002.

[15] E. B. Hooper, "Plasma detachment from a magnetic nozzle," J. Propulsion Power, vol. 9, p. 757, 1993

[16] S. A. Cohen, N. S. Siefert, S. Stange, R. F. Boivin, E. E. Scime, and F. M. Levinton, "Ion acceleration in plasmas emerging from a heliconheated magnetic-mirror device," Phys. Plasmas, vol. 10, p. 2593, 2003

[17] A. V. Arefiev and B. N. Breizman, "Theoretical components of the VASIMR plasma propulsion concept," Phys. Plasmas, vol. 11, p. 2942, 2004.

[18] M. D. Carter, F. W. Baity, Jr., G. C. Barber, R. H. Goulding, D. O. Spareks, K. F. White, and E. F. Jaeger, "Comparing experiments with modeling for light ion helicon plasma sources," Phys. Plasmas, vol. 9, p. 5097, 2002.

[19] A. Ilin, F. Chang Diaz, J. Squire, and M. D. Carter, "Plasma heating simulation in the VASIMR system," AIAA, 2005.

[20] C. Charles and R. W. Boswell, "Current-free double-layer formation in a high-density helicon discharge," Appl. Phys. Lett., vol. 82, p. 1356, 2003

[21] L. R. Block, “A double layer review," Astrophysics and Space Science, vol. 55, p. 59, 1978.

[22] N. Hershkowitz, "Review of recent laboratory double layer experiments," Space Sci. Rev., vol. 41, p. 351, 1985.

[23] M. A. Raadu, "The physics of double layers and their role in astrophysics," Phys. Rep., vol. 178, p. 25, 1989.

[24] G. Hairapetian and R. Stenzel, "Observation of a stationary, currentfree double layer in a plasma," Phys. Rev. Lett., vol. 65, p. 175, 1990.

[25] — - "Particle dynamics and current-free double layers in an expanding, collisionless, two-electron-population plasma," Phys. Fluids $B$, vol. 3, p. 899, 1991.

[26] R. Schrittwieser, I. Axnas, T. Carpenter, and S. Torven, "Observation of double layers in a convergent magnetic field," IEEE Trans. Plasma Sci., vol. 20, no. 6, pp. 607-613, Dec. 1992.

[27] K. Sato and F. Miyawaki, "Formation of presheath and current-free double layer in a twoelectron-temperature plasma," Phys. Fluids B, vol. 4, p. $1247,1992$.

[28] N. Plihon, C. S. Corr, and P. Chabert, "Double layer formation in the expanding region of an inductively coupled electronegative plasma," Appl. Phys. Lett., vol. 86, p. 091501, 2005.

[29] X. Sun, A. M. Keesee, C. Biloiu, E. E. Scime, A. Meige, C. Charles, and R. W. Boswell, "Observations of ion-beam formation in a current-free double layer," Phys. Rev. Lett., vol. 95, p. 025004, 2005.

[30] X. Sun, E. Scime, M. Miah, S. Cohen, and F. Skiff, "Measurement of asymmetric optical pumping of ions accelerating in a magnetic field gradient," Phys. Rev. Lett., vol. 93, p. 23502, 2004.

[31] G. D. Severn, D. A. Erlich, and R. McWilliams, "Argon ion laser-induced fluorescence with diode lasers," Rev. Sci. Instrum., vol. 69, p. 10, 1998.

[32] R. F. Boivin and E. E. Scime, "Laser induced fluorescence in Ar and He plasmas with a tunable diode laser," Rev. Sci. Instrum., vol. 74, p. 4352, 2003.
[33] R. L. Kurucz and B. Bell "1995 atomic line data," Smithsonian Astrophysical Observatory, CD-ROM No.23 1995.

[34] S. Sasaki, S. Takamura, S. Watanabe, S. Masuzaki, T. Kato, and K. Kadota, "Helium I line intensity ratios in a plasma for the diagnostics of fusion edge plasmas," Rev. Sci. Instrum., vol. 67, p. 3521, 1996.

[35] R. F. Boivin, "Zeeman splitting for LIF transitions and de-convolution technique to extract ion temperature," EPAPS Deposited Document E-PHPAEN-10-003306, 2003.

[36] X. Sun, S. A. Cohen, M. Miah, and E. Scime, "On-axis parallel ion speeds near mechanical and magnetic apertures in a helicon plasma devices," Phys. Plasmas, vol. 12, p. 103509, 2005

[37] K. Shiraishi and S. Takamura, "Sheath formation in the SOL plasma with energetic electrons," J. Nucl. Materials, vol. 176 and 177, p. 251, 1990.

[38] M. Cercek and T. Gyergyek, "Collector floating potentials in a discharge plasma," J. Phys. D, vol. 34, p. 330, 2001.

[39] F. F. Chen and D. D. Blackwell, "Upper limit to Landau damping in helicon discharges," Phys. Rev. Lett., vol. 82, p. 2677, 1999.

[40] R. F. Boivin, J. L. Kline, and E. E. Scime, "Electron temperature measurement by a helium line intensity ratio method in helicon plasmas," Phys. Plasmas, vol. 8, p. 5303, 2001

[41] S. M. Tysk, C. Mark Denning, J. E. Scharer, and K. Akhtar, "Optical, wave measurements, and modeling of helicon plasmas for a wide range of magnetic fields," Phys. Plasmas, vol. 11, p. 878, 2004.

[42] R. T. S. Chen and N. Hershkowitz, "Multiple electron beams generated by a helicon plasma discharge," Phys. Rev. Lett., vol. 80, p. 4677, 1998.

[43] P. Zhu and R. W. Boswell, "Observation of nonthermal electron tails in an rf excited argon magnetoplasma," Phys. Fluids B, vol. 3, p. 869, 1991.

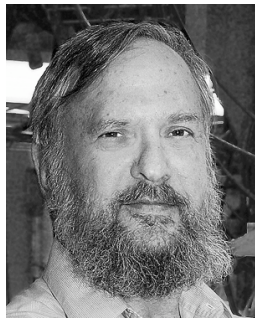

Samuel A. Cohen received the B.S. and Ph.D. degrees from the Massachusetts Institute of Technology (MIT), Cambridge, in 1968 and 1973, respectively.

$\mathrm{He}$ is a Lecturer with rank of Professor in the Department of Astrophysical Sciences and Director of the Program in Plasma Science and Technology at Princeton University. His research is on plasma physics for controlled fusion, with focus on the reversed-field configuration (FRC), an innovative confinement concept that holds much promise for clean compact fusion reactors. He has published over two hundred refereed papers, co-edited three books, and co-authored two books. For 13 years, he served as the Resident Associate Editor of Physics of Plasmas.

Dr. Cohen has won MIT's Goodwin Medal for Distinguished Teaching and is a fellow of the American Physical Society.

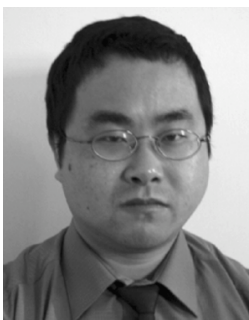

Xuan Sun received the M.S. degree from University of Science and Technology of China, Hefei, in 2000, and the $\mathrm{Ph} . \mathrm{D}$. degree in plasma physics for the experimental study of ion acceleration, asymmetric optical pumping, and low frequency waves in two expanding helicon plasma sources, from West Virginia University, Morgantown, in 2005.

His current research interests include the study of magnetospheric ion temperature evolution during geomagnetic storms and development of a fast temporal-resolved laser-induced fluorescence mea-

surement.

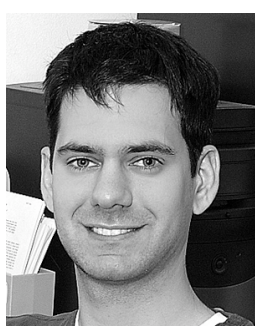

Nathaniel M. Ferraro is currently working toward the Ph.D. degree in the Astrophysical Sciences Program at Princeton University, Princeton, NJ. 


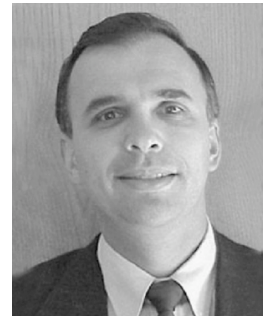

Earl E. Scime received the B.S. degrees in physics and applied mathematics from Florida State University, Tallahassee, in 1987, and the Ph.D. degree in experimental plasma physics from the University of Wisconsin, Madison, in 1992.

From 1992 to 1994, he was a Department of Energy Distinguished Postdoctoral Fellow in the Space Plasma Physics group at Los Alamos National Laboratory, Los Alamos, NM. In 1995, he moved to West Virginia University, Morgantown, where he is now Professor and Chair of Physics. His research interests include charge exchange imaging of high-temperature plasmas, the mechanisms of ion heating in space and laboratory plasmas, spacecraft charging, helicon plasma source development, laser induced fluorescence diagnosis of laboratory plasmas, plasma thrusters, electric double layers, plasma processing, coherent microwave scattering diagnosis of laboratory plasmas, and the theory of instabilities driven by flow shear and thermal anisotropy. He is an author or coauthor of over 100 refereed publications.

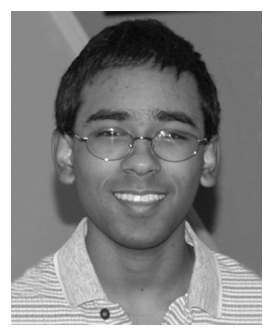

Mahmood Miah received the B.S. degree in physics from the University of California, Irvine, in 2002. He is current working toward the Ph.D. degree in computational plasma physic in the Department of Astrophysical Sciences, Program in Plasma Physics, at Princeton University, Princeton, NJ

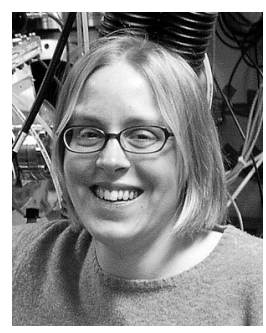

Sy Stange received the M.S. degree in astrophysical sciences from Princeton University, Princeton, NJ. $\mathrm{He}$ is currently working toward the Ph.D. degree in the Nuclear Engineering Program at the University of Michigan, Ann Arbor.

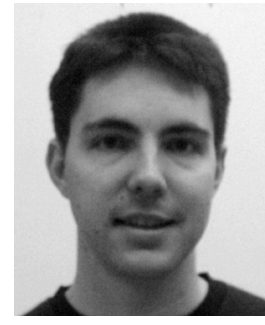

Nicholas S. Siefert was born in 1980. He received the B.S.E. degree from Princeton University, Princeton, $\mathrm{NJ}$, in 2002

He is presently with the Air Force Research Laboratory, Wright Patterson Air Force Base, OH. His current areas of research are double layers and shockwave interactions with plasma.

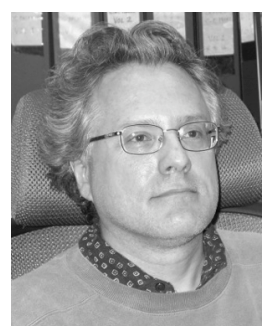

Robert F. Boivin received the B.Sc. degree from the University of Montreal, Montreal, QC, Canada, in 1980, and the M.Sc. and Ph.D. degrees, INRS-Energy and Materials, University of Quebec, Montreal, QC, Canada, 1985 and 1992, respectively. His M.Sc. thesis was on a construction and characterization of a Z-pinch plasma source. His Ph.D. dissertation featured the development of a new Pulsed Laser Induced Desorption (PLID) technique to measure hydrogen content in materials is presently used to measure fluxes in large toroidal device.

He is presently an Assistant Professor at the Physics Department, Auburn University. He was a Postgraduate Researcher at the Plasma Research Group, Physics Department, West Virginia University (WVU), Morgantown. He was a Research Associate at the Atomic and Molecular Collision Research Element; Earth and Space Sciences Division. Jet Propulsion Laboratory (JPL). He was a Postgraduate Researcher at the Fusion Energy Research Program, University of California, San Diego (UCSD). He was a Postgraduate Researcher at the Institute of Plasma and Fusion Research, University of California, Los Angeles (UCLA). He has extensive experience in plasma physics laboratory involving laser spectroscopy, spectroscopy, Langmuir probe, microwave, and many other innovative diagnostics. As an experimentalist, he has been designing, constructing, and operating laboratory plasma sources for the past 15 years. He has work on a Z-pinch, a Tokamak (TdeV), a reversed arc device (PISCES), and three different helicon plasma sources. During his many years of postdoctoral research, he has acquired an extended scientific expertise in plasma, plasma-wall interaction and plasma diagnostics. He developed new technique to measure plasma parameters and atomic process in plasmas. He also worked on the field of atomic and molecular physics by measuring electron impact ionization cross sections by means of electron and molecular crossed beams. He has a strong expertise in laser (pulsed and CW), laser instrumentation, spectroscopy (UV and visible), laser-surface interactions, calibration techniques, plasma and plasma diagnostics, ion implantation, and vacuum science. 